\title{
Functional Communication Scale
}

National Cancer Institute

\section{Source}

National Cancer Institute. Functional Communication Scale. NCI Thesaurus. Code C121299.

A rating scale included in the Behavior Assessment System for Children that measures the subject's ability to express ideas and communicate in a way others can easily understand. 